\section{Van Gogh: Manie und Melancholie}

\author{
Ein Porträt
}

Manfred Clemenz. Van Gogh: Manie und

Melancholie: Ein Porträt. Köln: Böhlau; 2020,

450 Seiten, gebunden, 55,00 Euro,

ISBN 9783412515942

Trotz der glühenden Farben und ekstatischen Formen: Für van Gogh ist Kunst Mühsal und Leiden. Zugleich ist sie für ihn die einzige Therapie gegen Melancholie. Für inn ist das Kunstschaffen also Krankheit und Therapie zugleich. Tiefste Melancholie und ekstatischer Schaffensrausch wechseln bei ihm einander ab, wobei das Leiden für van Gogh eine Voraussetzung für seine Kreativität darstellt. Es handelt sich für ihn dabei um einen höheren Bewusstseinszustand, den er für seine künstlerische Tätigkeit unbedingt braucht und daher häufig selbst herbeiführt: durch Nahrungsentzug, durch Intoxikation, durch Selbstkasteiung.

Kunst ist für van Gogh aber auch Therapie durch mühsame Arbeit, zugleich die Möglichkeit, sich seiner eigenen Bedeutung als Teil einer „Wiedergeburt der Kunst“ zu vergewissern. Entwertung der eigenen Person und grandiose Selbsterhöhung stehen sich bei ihm dabei unmittelbar gegenüber. Der Autor Manfred Clemenz (Soziologe, Psychotherapeut und Kunsthistoriker) stützt sich bei seiner Metaanalyse des außerordentlich kontrovers diskutierten Themas von van Goghs Krankheit vor allem auf Vincents eigene Korrespondenz, auf historisch belegte Fakten und auf die relevante Sekundärliteratur. Einigermaßen sicher können wir aus den zeitgenössischen Beschreibungen und Diagnosen nur entnehmen, dass van Gogh in seinen letzten beiden Jahren unter epileptoiden Anfällen, extremen Stimmungsschwankungen, Halluzinationen und Amnesien litt.

Clemenz gelingt es in dieser fundierten und inspirierenden Metaanalyse anhand ausgewählter Texte jedoch, ganz neue Sichtweisen auf van Goghs Erkrankungen zu eröffnen. Auf diese Weise wird das Interpretationsspektrum bezüglich der Wechselbeziehungen zwischen den Krankheiten van Goghs und seinem künstlerischen Schaffen erheblich erweitert. Van Gogh verortet sich selbst zwischen Manie und Melancholie, dabei unterscheidet er bei sich eine destruktive und eine kreative, aktive Melancholie. Intuitiv bezieht er sich damit auf eine Diagnose, die bereits seit der Antike bekannt ist und einen Gemütszustand beschreibt, der zwischen Depression und Manie schwankt. In Wirklichkeit jedoch muss es sich bei van Gogh um einen viel komplexeren und mehrdimensionalen Wirkungszusammenhang zwischen seinen Krankheiten und seinem künstlerischen Schaffen gehandelt haben, wie Clemenz in seinem äußerst informativen Buch sehr anschaulich aufzeigen kann.

\section{Christian Hellweg, Frankfurt am Main}

\section{Muskeln, Faszien und Schmerz}

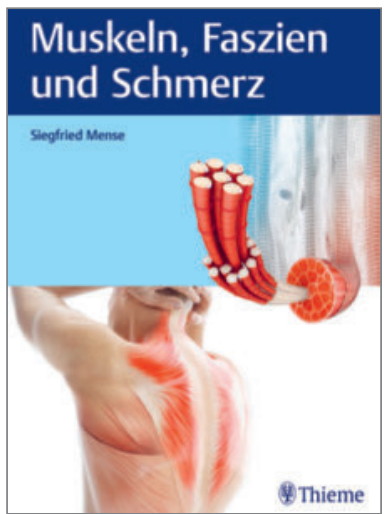

Siegfried Mense. Muskeln, Faszien und Schmerz. Stuttgart: Thieme Verlag; 2021, 179 Seiten, 192 Abbildungen, 79,99Euro, ISBN 9783132426610

Der emeritierte Professor für Anatomie an der Universität Heidelberg richtet sich mit seinem Buch nach eigenen Worten „an alle Berufsgruppen, die sich mit dem Bewegungsapparat und dessen schmerzhaften Störungen befassen“. Neben Physiotherapeuten, verwandten Berufen und Ärzten hat er auch Betroffene im Blick, die „mehr über die Funktion des motorischen Systems wissen möchten“. Ein schönes Ansinnen, doch für diese potenziellen Leser ist das Buch aus meiner Sicht trotz ausführlicher Erklärungen und didaktisch hervorragend aufbereitetem Inhalt zu fachspezifisch. Für die andere Zielgruppe, und dazu zählen auch Sportwissenschaftler und Trainer, die über ein grundlegendes anatomisches Wissen verfügen, bietet das Werk viele Informationen zu einem spannenden Thema von hoher klinischer Relevanz.
Ausführlich erklärt Mense die neuroanatomischen und neurophysiologischen Grundlagen des tiefsomatischen Schmerzes, der sich wesentlich vom Hautschmerz unterscheidet. Weil letzterer historisch die Grundlage für die Schmerzforschung war und viele Kenntnisse wie selbstverständlich auf Muskelschmerzen übertragen werden, beschreibt der Autor die Besonderheiten des Muskelschmerzes. Auch die funktionellen Eigenschaften der Muskulatur sowie Aufbau, Funktion und Innervation der Faszien werden ausführlich dargestellt. Spannend wird es, wenn der Autor die unterschiedlichen schmerzhaften Störungen faszialer Gewebe erklärt und dies anhand typischer Schmerzsymptome wie dem medialen Tibiakanten- oder dem ISG-Syndrom veranschaulicht. Im Kapitel „Schmerzhafte funktionelle Störungen der Muskulatur“ werden nicht nur Prellungen, Zerrungen und Faserrisse besprochen, auch muskuläre Dysbalancen, neurogene (Restless-legs-Syndrom), durchblutungsbedingte (Claudicatio intermittens, Kompartment-Syndrom) sowie sonstige funktionelle Störungen und Myositis werden abgehandelt.

Alles in allem bietet das Buch von Mense einen fundierten Überblick über klinisch relevante Schmerzphänomene, und das alles auf Grundlage ausführlicher anatomischer Beschreibungen. Wer sich mit der Physiologie der Schmerzentstehung beschäftigen möchte und an einem Überblick über Schmerzen des Bewegungssystems interessiert ist, ist mit dem Kauf dieses Buches gut beraten. Zwar ist die meiste zitierte Literatur schon etwas älter, aber das liegt wohl daran, dass der Autor jahrzehntelang zum Thema Schmerz geforscht hat und, wie er im Vorwort verrät, das Buch geschrieben hat, weil ihm in vielen Diskussionen und Fragen nach seinen Vorträgen bei Kongressen klar geworden ist, „dass vielen der Zuhörer das grundlegende Wissen über die Neuroanatomie des Bewegungsapparates und über Schmerzmechanismen fehlte“. Da der Autor nicht selbst Therapeut ist, mangelt es ihm zwar an der Erfahrung eines Praktikers. Da er aber keiner therapeutischen „Schule“ angehört, ist seine Ausarbeitung frei von Dogmen - ein Faktum, das man nicht unterschätzen sollte.

Johannes Ermel, Ettlingen 